\title{
AVALIAÇÃO DA BIODEGRADABILIDADE DE EFLUENTES TÊXTEIS DO BENEFICIAMENTO SECUNDÁRIO DE MALHAS DE POLIÉSTER TRATADOS COM REAGENTE DE FENTON
}

\author{
Dângelle M. Oliveira* e Mônica M. D. Leão \\ Departamento de Engenharia Sanitária e Ambiental, Universidade Federal de Minas Gerais, Av. Antonio Carlos, 6627, 4ํandar,
} 31270-901 Belo Horizonte - MG, Brasil

Recebido em 10/9/08; aceito em 28/5/09; publicado na web em 10/11/09

\begin{abstract}
BIODEGRADABILITY EVALUATION OF TEXTILE EFFLUENTS FROM THE SECUNDARY PROCESS OF POLYESTER FIBERS TREATED WITH FENTON'S REAGENT. From the environmental point of view, the textile sector is outstanding for the generation of large amounts of biorecalcitrant effluents. In this paper the textile effluent biodegradability, both before and after its treatment with Fenton's Reagent, were compared by means of biologic tests. These tests showed that the Fenton treatment lowered the biodegradabilty of practically all tested effluents, except one specific effluent from a scouring bath of polyester fibers, which achieved a 93\% COD removal. This removal was due to a significant phase separation (oil/water).
\end{abstract}

Keywords: biodegradability; textile effluent; Fenton's reagent.

\section{INTRODUÇÃO}

Do ponto de vista ambiental, o setor têxtil tem se destacado por suas atividades consideravelmente poluidoras. Os efluentes têxteis caracterizam-se tanto pelo grande volume gerado quanto pela alta DQO e baixa biodegradabilidade devida ao uso intenso de água e de diversas substâncias químicas orgânicas e inorgânicas.

Geralmente, a redução da carga poluidora deste tipo de efluente aos níveis permitidos pela legislação ambiental para descarte em corpos d'água, exige a implementação de sistemas de tratamento de efluentes integrados (químico/biológico) que apresentam alto custo de instalação e operação. O sistema de tratamento químico mais comumente utilizado no setor têxtil é o de coagulação química, já o biológico é o de lodos ativados. Dessa forma, faz-se necessário o estudo de alternativas que possam melhorar a biodegradabilidade deste tipo de efluente, o que poderia reduzir o custo de instalação de ETE (Estações de Tratamento de Efluentes) por lodos ativados (opção de tratamento destrutivo mais barata).

Dentre os Processos Oxidativos Avançados (POA) existentes (sistemas $\mathrm{UV} / \mathrm{TiO}_{2}, \mathrm{UV} / \mathrm{O}_{3}, \mathrm{H}_{2} \mathrm{O}_{2} / \mathrm{UV}, \mathrm{Fe}(\mathrm{II}) / \mathrm{H}_{2} \mathrm{O}_{2}$ ou $\mathrm{Fe}(\mathrm{III}) / \mathrm{H}_{2} \mathrm{O}_{2}$ ), muitos autores ${ }^{1-5}$ têm apontado o Reagente de Fenton como uma tecnologia alternativa para o tratamento de efluentes têxteis, visando a sua oxidação parcial, de forma a gerar substâncias intermediárias mais biodegradáveis, ou completa, resultando em gás carbônico, água e alguns sais inorgânicos.

Outros autores ${ }^{6}$ indicaram a possibilidade do uso dessa tecnologia como pré-tratamento de uma parcela dos efluentes têxteis após testar o uso do Reagente de Fenton como etapa complementar ao tratamento biológico por lodos ativados.

$\mathrm{O}$ Reagente de Fenton ( $\mathrm{Fe}(\mathrm{II}) / \mathrm{H}_{2} \mathrm{O}_{2}$ ) é obtido por uma mistura de peróxido de hidrogênio e um sal de ferro (II) (geralmente sulfato ferroso) em meio ácido, permitindo a formação dos radicais hidroxila $(\bullet \mathrm{OH})$, oxidante forte e não seletivo $\left(\mathrm{E}^{\circ}=2,8 \mathrm{~V}\right)$.

As Equações químicas 1 a $4{ }^{7}$ ilustram o mecanismo das reações de decomposição catalítica do $\mathrm{H}_{2} \mathrm{O}_{2}$ por sais de ferro, em meio ácido:

$\mathrm{Fe}^{2+}+\mathrm{H}_{2} \mathrm{O}_{2} \rightarrow \mathrm{Fe}^{3+}+\mathrm{OH}^{-}+\cdot \mathrm{OH}$

\footnotetext{
*e-mail: dangellem@yahoo.com.br
}

$$
\begin{aligned}
& \mathrm{RH}+\bullet \mathrm{OH} \rightarrow \mathrm{R} \bullet+\mathrm{H}_{2} \mathrm{O} \\
& \mathrm{R} \bullet+\mathrm{Fe}^{3+} \rightarrow \mathrm{R}++\mathrm{Fe}^{2+} \\
& \mathrm{Fe}^{2+}+\bullet \mathrm{OH} \rightarrow \mathrm{Fe}^{3+}+\mathrm{OH}^{-}
\end{aligned}
$$

Após a geração da molécula com carbocátion $(\mathrm{R}+)$, esta pode ser oxidada completamente a gás carbônico e água ou parcialmente, em subprodutos orgânicos.

A utilização do Reagente de Fenton em efluentes biorrecalcitrantes apresenta grandes vantagens em relação aos tratamentos físicoquímicos convencionais, tais como menor geração de lodos, por se tratar de um sistema destrutivo, o que evita a alocação de recursos para tratamento e disposição de resíduos sólidos e a possibilidade de destruição de substâncias químicas persistentes.

Apesar da grande eficiência na remoção completa de poluentes orgânicos, a utilização do Reagente de Fenton é economicamente inviável no caso de grandes volumes de efluentes, havendo a necessidade de sua associação a outros tipos de tratamentos físico-químicos ou biológicos e/ou tratamento de parte do efluente bruto, ou seja, somente a fração realmente concentrada, biorrecalcitrante e tóxica.

Nesse trabalho foi comparada a biodegradabilidade de efluentes têxteis, provenientes das etapas de purga e tingimento de tecidos sintéticos, antes e após seu tratamento com Reagente de Fenton. Os experimentos foram realizados utilizando-se concentrações de Reagente de Fenton menores que as usuais com os objetivos de favorecer a oxidação incompleta das substâncias que compõem o efluente, promovendo a formação de compostos intermediários menos tóxicos e menos biorrecalcitrantes, passíveis de serem completamente oxidados posteriormente, em um sistema de lodos ativados comum e de disponibilizar uma tecnologia alternativa de menor custo, capaz de melhorar a biodegradabilidade deste tipo de efluente.

\section{PARTE EXPERIMENTAL}

Os efluentes têxteis utilizados nesse experimento são oriundos das etapas de purga e tingimento de tecidos, apresentando composição bastante variada. Estes efluentes foram selecionados a partir de um trabalho mais amplo, ${ }^{8}$ no qual foram apontados como sendo 
os menos biodegradáveis e mais concentrados, em termos de DQO, dentre aqueles gerados na indústria têxtil estudada.

Os efluentes foram coletados em uma indústria têxtil de acabamento de malhas, de médio porte, localizada na região metropolitana de Belo Horizonte, cuja produção está voltada principalmente para tecidos sintéticos $(65 \%)$.

Os ensaios de tratabilidade com Reagente de Fenton foram realizados em Jar Test seguindo as etapas de acidificação do efluente com ácido sulfúrico concentrado até $\mathrm{pH}=3$, dosagem de $\mathrm{FeSO}_{4} \cdot 7 \mathrm{H}_{2} \mathrm{O}$ e $\mathrm{H}_{2} \mathrm{O}_{2}$ em $10 \mathrm{~min}$, agitação lenta (100 rpm) durante $1 \mathrm{~h}$, sedimentação por $0,5 \mathrm{~h}$, coleta de uma alíquota do sobrenadante e posterior neutralização, com $\mathrm{NaOH} 0,1 \mathrm{M}$, sedimentação por 15 min e coleta de amostra para ensaio biológico e análises de DQO, DBO e peróxido de hidrogênio residual.

A eficiência do tratamento foi mensurada por meio da quantificação da remoção total de DQO, ou seja, promovida pela coagulação e mineralização juntas.

Baseado em um estudo realizado anteriormente ${ }^{6}$ para efluentes têxteis, a relação $\left[\mathrm{H}_{2} \mathrm{O}_{2}\right]:\left[\mathrm{Fe}^{2+}\right]$ utilizada foi de 5:1 (em massa). Já a relação DQO: $\left[\mathrm{H}_{2} \mathrm{O}_{2}\right]:\left[\mathrm{Fe}^{2+}\right]$ foi de 40:5:1, 50:5:1 e 60:5:1, tendo sido definidas de forma que o custo com reagentes por metro cúbico de efluente tratado não inviabilizasse uma possível utilização desse sistema em escala real.

Também foram realizados testes para averiguar a influência da temperatura na eficiência da reação.

A avaliação da biodegradabilidade dos efluentes foi feita por meio da variação da relação DQO/DBO e por ensaios biológicos. ${ }^{9}$ Esses ensaios (Figura 1) constituíram-se de 3 provetas de $1000 \mathrm{~mL}$, sendo que duas continham inóculo aclimatado (coletado na ETE biológica da empresa) e o efluente em estudo (antes e após o tratamento com Fenton) e uma apenas inóculo (controle). Esses sistemas foram mantidos sob aeração, com aerador de aquário, por até 7 dias. A remoção da carga orgânica pelos micro-organismos foi monitorada no $1^{\circ}, 4^{\circ}$ e $7^{\circ}$ dia de ensaio por meio da redução da DQO (Demanda Química de Oxigênio) e, em alguns casos, do COD (Carbono Orgânico Dissolvido).

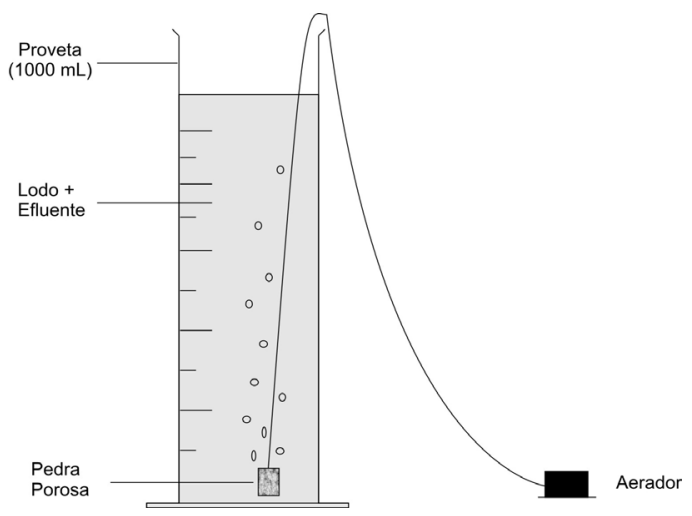

Figura 1. Montagem utilizada nos ensaios biológicos

Em todos os ensaios foi utilizada sempre uma relação volumétrica de inóculo/efluente (tratado com Fenton ou bruto) de 1:1.

O inóculo utilizado em cada bateria de ensaios foi caracterizado por meio do seu teor de SSV (Sólidos Suspensos Voláteis).

Em alguns casos também foi feita, no $1^{\circ}$ dia de ensaio, a leitura da condutividade elétrica das soluções (inóculo mais efluente ou inóculo puro), com o objetivo de estabelecer uma correlação desta com a remoção de DQO e/ou COD.

A interferência exercida pelo peróxido de hidrogênio nas análises de DQO foi eliminada por meio da determinação da sua concentração na amostra pelo método iodométrico ${ }^{10}$ e posterior determinação de sua $\mathrm{DQO}^{11}$ segundo a fórmula:

$\mathrm{DQOH}_{2} \mathrm{O}_{2}(\mathrm{mg} / \mathrm{L})=-4,06 \times 10^{-5} \cdot\left[\mathrm{H}_{2} \mathrm{O}_{2}\right]^{2}+0,4706 \cdot\left[\mathrm{H}_{2} \mathrm{O}_{2}\right]$

na qual: $\mathrm{DQOH}_{2} \mathrm{O}_{2}-\mathrm{DQO}$ do peróxido de hidrogênio, em mg de $\mathrm{O}_{2} / \mathrm{L} ;\left[\mathrm{H}_{2} \mathrm{O}_{2}\right]$ - concentração de peróxido de hidrogênio residual na amostra, em mg/L.

Todas as análises (DBO, DQO, COD e SSV) foram executadas de acordo com métodos padronizados, ${ }^{12}$ sendo que as análises de DQO foram realizadas pelo método colorimétrico com refluxo fechado e as de DBO pelo método iodométrico.

Todos os reagentes utilizados são de grau de pureza p.a..

\section{RESULTADOS E DISCUSSÃO}

\section{Ensaios com Reagente de Fenton}

A Tabela 1 sintetiza todos os ensaios realizados com o Reagente de Fenton e os diferentes efluentes provenientes das etapas de purga e tingimento de tecido sintético.

Em todos os ensaios realizados com efluentes de tingimento, a remoção em termos de DQO foi pequena, exceto no experimento 1. Neste ensaio foi observada razoável formação de flocos, o que indica que a maior parte da remoção foi devida à coagulação química da matéria orgânica em suspensão e não à sua conversão a gás carbônico e água (oxidação completa).

Durante todos os ensaios (efluentes de tingimento ou purga) foi possível notar que há ocorrência de coagulação química poucos minutos após o início do teste, o que contribui para a remoção de DQO.

A coagulação química associada ao Reagente de Fenton é explicada pela geração de íons $\mathrm{Fe}^{3+}$ (Equações 1 e 4) que, ao reagirem com íons hidróxido, dão origem a complexos hidroxo-férricos: ${ }^{4,13}$

$\left[\mathrm{Fe}\left(\mathrm{H}_{2} \mathrm{O}\right)_{6}\right]^{3+}+\mathrm{H}_{2} \mathrm{O} \rightleftharpoons\left[\mathrm{Fe}\left(\mathrm{H}_{2} \mathrm{O}\right)_{5} \mathrm{OH}\right]^{2+}+\mathrm{H}_{3} \mathrm{O}^{+}$

$\left[\mathrm{Fe}\left(\mathrm{H}_{2} \mathrm{O}\right)_{5} \mathrm{OH}\right]^{2+}+\mathrm{H}_{2} \mathrm{O} \rightleftharpoons\left[\mathrm{Fe}\left(\mathrm{H}_{2} \mathrm{O}\right)_{4}(\mathrm{OH})_{2}\right]^{2+}+\mathrm{H}_{3} \mathrm{O}^{+}$

que entre $\mathrm{pH} 3$ e 7 se tornam:

$2\left[\mathrm{Fe}\left(\mathrm{H}_{2} \mathrm{O}\right)_{5} \mathrm{OH}\right]^{2+} \rightleftharpoons\left[\mathrm{Fe}\left(\mathrm{H}_{2} \mathrm{O}\right)_{8}(\mathrm{OH})_{2}\right]^{4+}+2 \mathrm{H}_{2} \mathrm{O}$
$\left[\mathrm{Fe}\left(\mathrm{H}_{2} \mathrm{O}\right)_{8}(\mathrm{OH})_{2}\right]^{4+}+\mathrm{H}_{2} \mathrm{O} \rightleftharpoons\left[\mathrm{Fe}_{2}\left(\mathrm{H}_{2} \mathrm{O}\right)_{7}(\mathrm{OH})_{3}\right]^{3+}+\mathrm{H}_{3} \mathrm{O}^{+}$

$\left[\mathrm{Fe}_{2}\left(\mathrm{H}_{2} \mathrm{O}\right)_{7}(\mathrm{OH})_{3}\right]^{3+}+\left[\mathrm{Fe}\left(\mathrm{H}_{2} \mathrm{O}\right)_{5} \mathrm{OH}\right]^{2+} \rightleftharpoons\left[\mathrm{Fe}_{2}\left(\mathrm{H}_{2} \mathrm{O}\right)_{7}(\mathrm{OH})_{4}\right]^{5+}+2 \mathrm{H}_{2} \mathrm{O}$

Esses complexos são os responsáveis pela capacidade de coagulação química do Reagente de Fenton.

A ação dual de oxidação e coagulação do Reagente de Fenton em processos de tratamento é citada por alguns autores, ${ }^{5}$ sendo que tanto o íon férrico quanto o íon ferroso são agentes coagulantes.

A eficiência de remoção de $\mathrm{DQO}^{7}$ devida à coagulação química ocasionada pela formação de complexos hidroxo-férricos pode, até mesmo, ser maior do que aquela exercida pela oxidação com peróxido de hidrogênio.

Como pode ser observado na Tabela 1, em praticamente todos os experimentos ocorreu um aumento da relação DQO/DBO. É importante lembrar que o valor da DBO não passou por correção para eliminar o efeito exercido pelo peróxido de hidrogênio (efeito redutor deste valor), portanto, este parâmetro deve ser considerado com cautela, sobretudo para aqueles experimentos que apresentaram um valor expressivo de peróxido residual. 
Tabela 1. Ensaios com Reagente de Fenton e efluentes das etapas de purga e tingimento de tecidos sintéticos

\begin{tabular}{|c|c|c|c|c|c|c|c|}
\hline Ensaio & Tecido / cor / etapa & DQO: $\mathrm{H}_{2} \mathrm{O}_{2}: \mathrm{Fe}^{2+}$ & $\begin{array}{l}\text { Peróxido residual } \\
\qquad \%)\end{array}$ & $\begin{array}{l}\text { DQO } \\
(\mathrm{mg} / \mathrm{L})\end{array}$ & $\begin{array}{c}\mathrm{DBO} \\
(\mathrm{mg} / \mathrm{L})\end{array}$ & DQO / DBO & $\begin{array}{l}\text { Remoção } \\
\text { DQO (\%) }\end{array}$ \\
\hline \multirow{4}{*}{1} & \multirow{4}{*}{$\begin{array}{c}100 \% \text { Poliéster / escuro / } \\
\text { Tingimento }\end{array}$} & Efluente Bruto & - & 5129 & 1255 & 4 & - \\
\hline & & $40: 5: 1$ & 17 & 1199 & - & - & 77 \\
\hline & & $50: 5: 1$ & 39 & 1666 & 232 & 7 & 68 \\
\hline & & $60: 5: 1$ & 51 & 3863 & 376 & 10 & 25 \\
\hline \multirow{4}{*}{2} & \multirow{4}{*}{$\begin{array}{c}100 \% \text { Poliéster / escuro / } \\
\text { Tingimento }\end{array}$} & Efluente Bruto & - & 6728 & 699 & 10 & - \\
\hline & & $40: 5: 1$ & 17 & 6121 & 389 & 16 & 9 \\
\hline & & $50: 5: 1$ & 9 & 6076 & 627 & 10 & 10 \\
\hline & & $60: 5: 1$ & 23 & 6125 & 773 & 8 & 9 \\
\hline \multirow{4}{*}{3} & \multirow{4}{*}{$\begin{array}{l}90 \% \text { Poliéster - } 10 \% \text { Elas- } \\
\text { tano / escuro / Tingimento }\end{array}$} & Efluente Bruto & - & 5998 & 1384 & 4 & - \\
\hline & & 40:5:1 & 6 & 5825 & 602 & 10 & 3 \\
\hline & & $50: 5: 1$ & 7 & 5991 & 814 & 7 & 0 \\
\hline & & $60: 5: 1$ & 6 & 5922 & 937 & 6 & 1 \\
\hline \multirow{4}{*}{4} & \multirow{4}{*}{$\begin{array}{l}90 \% \text { Poliéster - } 10 \% \text { Elas- } \\
\text { tano / escuro / Tingimento }\end{array}$} & Efluente Bruto & - & 4121 & 1052 & 4 & - \\
\hline & & $50: 5: 1$ & 15 & 3890 & 705 & 6 & 6 \\
\hline & & $60: 5: 1$ & 18 & 3937 & 782 & 5 & 4 \\
\hline & & $60: 5: 1\left(50^{\circ} \mathrm{C}\right)$ & 7 & 4017 & 816 & 5 & 3 \\
\hline \multirow{4}{*}{5} & \multirow{4}{*}{$\begin{array}{l}\text { 100\% Poliéster / } \\
\text { claro / Purga }\end{array}$} & Efluente Bruto & - & 10488 & 1620 & 6 & - \\
\hline & & $40: 5: 1$ & 46 & 816 & - & - & 92 \\
\hline & & $50: 5: 1$ & 45 & 780 & - & - & 93 \\
\hline & & $60: 5: 1$ & 46 & 757 & - & - & 93 \\
\hline \multirow{4}{*}{6} & \multirow{4}{*}{$\begin{array}{l}\text { 100\% Poliéster / } \\
\text { claro / Purga }\end{array}$} & Efluente Bruto & - & 10043 & 2371 & 4 & - \\
\hline & & 40:5:1 & 39 & 816 & - & - & 91 \\
\hline & & $50: 5: 1$ & 36 & 780 & - & - & 91 \\
\hline & & $60: 5: 1$ & 19 & 757 & - & - & 91 \\
\hline \multirow{4}{*}{7} & \multirow{4}{*}{$\begin{array}{l}\text { 100\% Poliéster / } \\
\text { claro / Purga }\end{array}$} & Efluente Bruto & - & 10408 & 2692 & 4 & - \\
\hline & & $40: 5: 1$ & 31 & 9825 & 2252 & 4 & 6 \\
\hline & & $50: 5: 1$ & 33 & 10001 & 1994 & 5 & 4 \\
\hline & & $60: 5: 1$ & 40 & 9916 & 2519 & 4 & 5 \\
\hline \multirow{4}{*}{8} & \multirow{4}{*}{$\begin{array}{l}90 \% \text { Poliamida - } 10 \% \text { Elas- } \\
\text { tano / branco / Purga }\end{array}$} & Efluente Bruto & - & 4259 & 1459 & 3 & - \\
\hline & & $15: 5: 1$ & 51 & 3543 & 625 & 6 & 17 \\
\hline & & $60: 5: 1$ & 56 & 4159 & 1154 & 4 & 2 \\
\hline & & $60: 5: 1\left(40^{\circ} \mathrm{C}\right)$ & 48 & 3987 & 893 & 4 & 6 \\
\hline \multirow{4}{*}{9} & \multirow{4}{*}{$\begin{array}{l}\text { 100\% Poliéster / } \\
\text { preto / Purga }\end{array}$} & Efluente Bruto & - & 5709 & 2316 & 2 & - \\
\hline & & $15: 5: 1$ & 31 & 3535 & 398 & 9 & 38 \\
\hline & & $60: 5: 1$ & 45 & 5366 & 1366 & 4 & 6 \\
\hline & & $60: 5: 1\left(40^{\circ} \mathrm{C}\right)$ & 26 & 5300 & 1661 & 3 & 7 \\
\hline
\end{tabular}

No ensaio 4 foi feito um teste para observar a influência exercida pela temperatura. $\mathrm{O}$ teste foi realizado a $50{ }^{\circ} \mathrm{C}$ e ao ser comparado com o experimento da mesma batelada com as mesmas características (relação 60:5:1) operado à temperatura ambiente $\left(26^{\circ} \mathrm{C}\right)$ é possível notar que ocorreu uma pequena redução na eficiência de remoção de DQO. Isso pode ser um indício da decomposição do peróxido de hidrogênio em oxigênio e água, o que é reforçado pelo menor valor de peróxido residual encontrado.

Os testes realizados com efluentes de purga apresentaram, de forma geral, o mesmo comportamento dos efluentes de tingimento no que diz respeito à relação DQO/DBO e eficiência na remoção de DQO.

Atenção especial deve ser dada aos ensaios 5 e 6. Esses dois efluentes, que possuem basicamente a mesma composição, sofreram grande remoção de DQO, obtida por meio de uma separação de fase expressiva ocorrida durante a reação com o Reagente de Fenton, mesmo utilizando-se concentrações menores de $\mathrm{H}_{2} \mathrm{O}_{2}$ e Fe ${ }^{2+}$ (relação 60:5:1).

Quando se observa o tipo de tecido e a composição dos efluentes testados nesses ensaios, é possível notar que eles apresentam a mesma composição daquele testado no ensaio 9, exceto pela presença do agente lubrificante "A", o qual é composto essencialmente por ésteres graxos, segundo informações do fabricante. Além disso, pode-se notar que o lubrificante " $A$ ” foi, provavelmente, responsável por aproximadamente $50 \%$ de toda a DQO do banho, uma vez que a DQO do efluente coletado para o ensaio 9 (sem lubrificante) é de $5.709 \mathrm{mg} / \mathrm{L}$, enquanto que as dos ensaios 5 e 6 (com lubrificante) são, respectivamente, 10.488 e $10.043 \mathrm{mg} / \mathrm{L}$. 
Tabela 2. Composição dos efluentes estudados

\begin{tabular}{|c|c|c|}
\hline Ensaio & DQO (mg/L) & Composição \\
\hline 1 & 5129 & $\begin{array}{l}\text { Lubrificante B, Dispersante A, Dispersante C, Barrilha, Soda cáustica, Redutor B, corantes dis- } \\
\text { persos amarelo S6GLSBC e turquesa SGL e água }\end{array}$ \\
\hline 2 & 6728 & $\begin{array}{l}\text { Dispersante A, Lubrificante C, Dispersante C, corantes dispersos amarelo E3 GFL, azul BTE } \\
\text { SE2RBC e laranja SERFL, soda cáustica, Redutor B e água }\end{array}$ \\
\hline 3 & 5998 & $\begin{array}{l}\text { Dispersante A, Lubrificante B, Dispersante B, Barrilha, corantes dispersos laranja SERFL, ver- } \\
\text { melho S3BS e vermelho SBS, soda cáustica, Redutor B, Emulsionante e água }\end{array}$ \\
\hline 4 & 4121 & $\begin{array}{l}\text { Dispersante A, Lubrificante D, corantes dispersos vermelho E2BL, bordô S2GFL e azul ELHC, } \\
\text { Redutor C, soda cáustica, Emulsionante e água }\end{array}$ \\
\hline 5 & 10488 & Emulgador, Solventes, Lubrificante A e água \\
\hline 6 & 10043 & Emulgador, Solventes, Lubrificante A e água \\
\hline 7 & 10408 & Emulgador, Solventes, Lubrificante B e água \\
\hline 8 & 4259 & Umectante, Detergente, Ácido Acético e água \\
\hline 9 & 5709 & Emulgador, Solventes e água \\
\hline
\end{tabular}

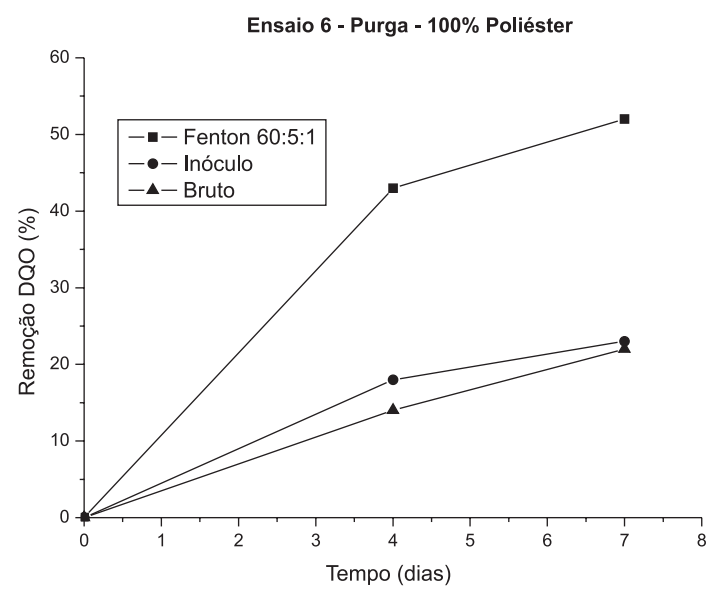

Figura 2. Ensaio biológico do efluente coletado e tratado com Reagente de Fenton no ensaio $6\left(S S V_{\text {inóculo }}=118 \mathrm{mg} / \mathrm{L}\right)$

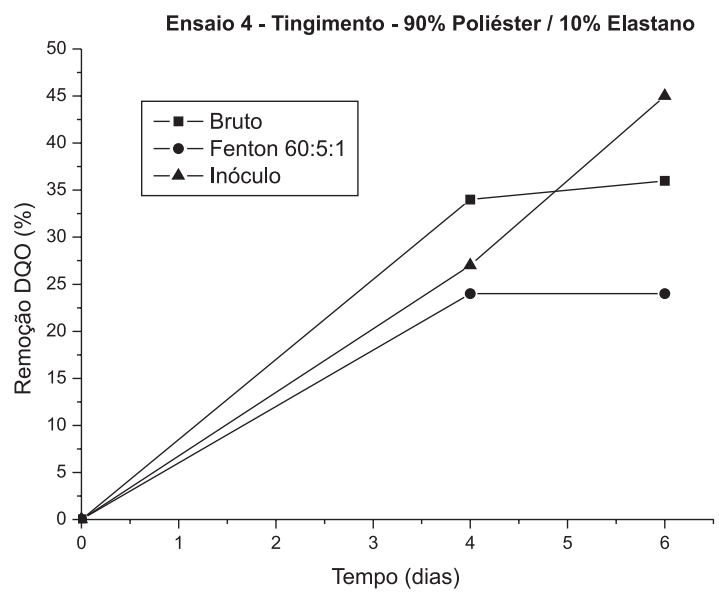

Figura 3. Ensaio biológico do efluente coletado e tratado com Reagente de Fenton no ensaio $4\left(S S V_{\text {inóculo }}=83 \mathrm{mg} / \mathrm{L}\right)$

Nos experimentos dos ensaios 8 e 9 foram realizados testes elevando-se a temperatura de tratamento para $40^{\circ} \mathrm{C}$. Isso levou a uma pequena melhoria na eficiência de remoção de DQO.

De forma geral, a quantidade de peróxido residual em solução

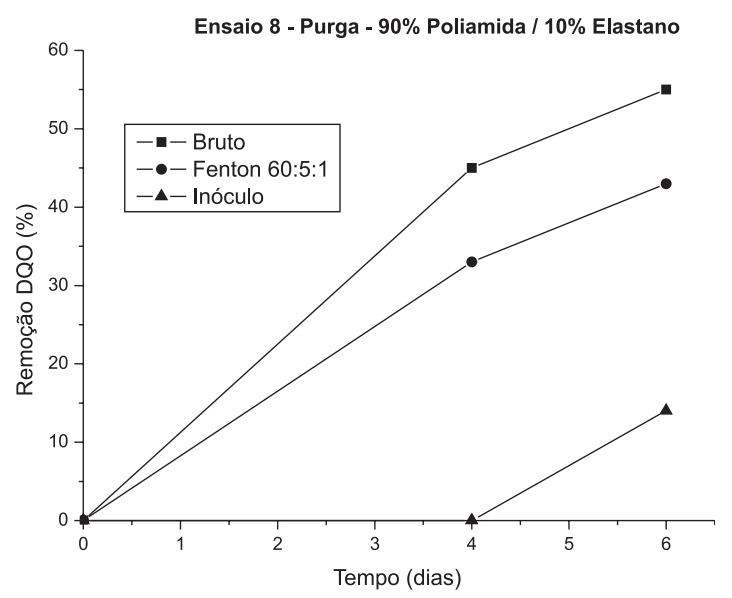

Figura 4. Ensaio biológico do efluente coletado e tratado com Reagente de Fenton no ensaio $8\left(S S V_{\text {inóculo }}=58 \mathrm{mg} / \mathrm{L}\right)$

manteve-se mais alta nos ensaios realizados com os banhos de purga do que nos de tingimento, o que pode ser um indício de que a reação de oxidação não ocorreu em sua total extensão. Uma possível explicação para isso pode ser a pouca solubilidade de algumas substâncias que se encontram emulsionadas na solução, tais como o óleo de urdume, extraído das fibras de poliéster durante o processo de purga, e agentes lubrificantes adicionados ao banho. A hidrofobicidade dessas substâncias pode ter levado a uma redução da superfície de contato entre os radicais hidroxila, gerados pelo sistema Fenton, e a superfície dessas moléculas, o que pode ter prolongado o tempo necessário para o completo processamento da reação e estabelecimento do equilíbrio químico.

Os ensaios 8 e 9 , conduzidos com uma quantidade maior de $\mathrm{H}_{2} \mathrm{O}_{2}$ e $\mathrm{Fe}^{2+}$ (15:5:1), não levaram a um aumento tão significativo na eficiência da reação. Isso pode ser mais um indício da menor reatividade dos efluentes provenientes de banhos de purga.

\section{Ensaios biológicos}

Os efluentes testados foram aqueles coletados e tratados com Fenton nos experimentos 4, 6 e 8. Em todos os ensaios o parâmetro utilizado para monitorar a remoção de matéria orgânica foi a DQO e, em dois casos (testes 8 e 9), também o COD. 
Tabela 3. Eficiência de remoção de COD e DQO e condutividade elétrica em efluentes no teste biológico

\begin{tabular}{lcccc}
\hline Efluente & Solução & $\begin{array}{c}\text { Remoção COD } \\
(\%)\end{array}$ & $\begin{array}{c}\text { Remoção DQO } \\
(\%)\end{array}$ & $\begin{array}{c}\text { Condutividade } \\
(\mathrm{mS} / \mathrm{cm})\end{array}$ \\
\hline Purga - 90\% & $50 \%$ bruto + 50\% Inóculo & 71 & 55 & 2,70 \\
Poliamida / 10\% & $50 \%$ Fenton + 50\% Inóculo & 32 & 43 & 3,27 \\
Elastano (ensaio 8) & $100 \%$ Inóculo & 1 & 14 & 5,63 \\
& $50 \%$ bruto + 50\% Inóculo & 68 & - & 3,40 \\
Purga - 100\% & $50 \%$ Fenton + 50\% Inóculo & 29 & - & 3,91 \\
Poliéster (ensaio 9) & $100 \%$ Inóculo & 1 & 11 & 5,76 \\
\hline
\end{tabular}

Como pode ser observado nas Figuras 2, 3 e 4, que apresentam a remoção de DQO no decorrer do ensaio, a remoção de DQO é quase sempre maior no efluente bruto, com exceção do tratamento com Fenton realizado no ensaio 6 (Purga - 100\% poliéster). Esse efluente sofreu uma redução de $93 \%$ na sua DQO, após o tratamento com Reagente de Fenton, devido a uma separação de fase substancial (óleo/água). Isso indica que praticamente toda a matéria orgânica foi eliminada, fazendo com que o efluente, provavelmente, ficasse livre de substâncias tóxicas e/ou biorrecalcitrantes.

A grande eficiência de remoção de DQO alcançada nos testes empregando esse efluente foi devida à presença de um produto lubrificante emulsionado, que provavelmente teve seu agente emulsionante oxidado pelo Reagente de Fenton. A remoção de matéria orgânica do efluente foi devida principalmente ao efeito coagulante dos íons Fe (III).

De forma geral, os ensaios biológicos confirmaram os resultados obtidos nas análises de DBO e indicaram que os efluentes, após passarem pelo tratamento com Reagente de Fenton, sofrem mudanças em suas composições de forma a torná-los menos vulneráveis à metabolização biológica.

Acredita-se que a redução da biodegradabilidade dos efluentes tratados pode ser devida à formação de compostos ainda mais tóxicos e/ou biorrecalcitrantes e ao grande aporte de sais provenientes das etapas de acidificação/neutralização e adição de $\mathrm{FeSO}_{4} \cdot 7 \mathrm{H}_{2} \mathrm{O}$. A toxicidade de efluentes tratados com Reagente de Fenton já foi registrada anteriormente por outros autores. ${ }^{14}$

Na Tabela 3 é feita uma comparação entre a condutividade elétrica dos diferentes sistemas ensaiados e a remoção de DQO e COD. Como esperado, os efluentes tratados com Fenton apresentam maior conteúdo de sais solubilizados, o que é expresso por sua elevada condutividade frente aos efluentes brutos.

O inóculo utilizado nos ensaios também apresentou elevada condutividade, o que pode ser explicado pelo uso intenso de sais (principalmente $\mathrm{NaCl}, \mathrm{Na}_{2} \mathrm{CO}_{3}$ ) e soda cáustica nos processos de tingimento, os quais são descartados com o efluente.

Analisando-se ainda a Tabela 3, pode-se observar que a eficiência de remoção de COD obtida após 6 dias de ensaio biológico apresenta a mesma tendência daquela encontrada tomando-se como parâmetro a DQO, ou seja, confirma o fato de que efluentes têxteis tratados com Reagente de Fenton são menos biodegradáveis ou são biodegradados de forma mais lenta.

Os dados levantados nessa pesquisa indicaram que o prétratamento de efluentes têxteis com Reagente de Fenton só melhora a sua biodegradabilidade em casos nos quais ocorre uma remoção significativa da matéria orgânica, a uma taxa de aplicação reduzida de 60:5:1 $\left(\left[\mathrm{H}_{2} \mathrm{O}_{2}\right]=835 \mathrm{mg} / \mathrm{L} ;\left[\mathrm{Fe}^{2+}\right]=167 \mathrm{mg} / \mathrm{L}\right)$, afim de evitar o aumento da concentração de eletrólitos na solução e em temperaturas em torno de $40{ }^{\circ} \mathrm{C}$.

\section{CONCLUSÕES}

Os ensaios biológicos realizados com efluentes e inóculo aclimatado, apesar de pouco sofisticados, mostraram-se mais adequados à avaliação da biodegradabilidade de efluentes tratados com Reagente de Fenton do que a relação DQO/DBO, devido à interferência exercida pelo peróxido de hidrogênio residual. O Reagente de Fenton não foi capaz de promover a melhoria da biodegradabilidade dos efluentes gerados nas etapas de purga e tingimento de malhas de poliéster, salvo alguns efluentes específicos provenientes de banhos de purga. Os tratamentos com Reagente de Fenton de alguns efluentes provenientes de banhos de purga de tecido $100 \%$ poliéster apresentaram uma eficiência na remoção de DQO de até $93 \%$, quando utilizadas baixas concentrações do Reagente de Fenton (relação DQO: $\mathrm{H}_{2} \mathrm{O}_{2}: \mathrm{Fe}^{2+}$ igual a 60:5:1 em massa). A eficiência de remoção de DQO por meio da utilização do Reagente de Fenton é melhorada em temperaturas em torno de 40 ${ }^{\circ} \mathrm{C}$, sendo que acima desse valor ocorre uma redução de eficiência.

Os ensaios foram realizados apenas em efluentes gerados no beneficiamento secundário de malhas de poliéster, não sendo prudente a sua generalização para efluentes do beneficiamento de outros substratos têxteis, ou para o efluente geral de uma indústria de beneficiamento de malhas.

\section{REFERÊNCIAS}

1. Rodriguez, M.; Sarria, V.; Esplugas, S.; Pulgarin, C.; J. Photochem. Photobiol., A 2002, 151, 129.

2. Pérez, M.; Torrades, F.; Doménech, X.; Peral, J.; Water Res. 2002, 36, 2703.

3. Balanosky, E.; Herrera, F.; Lopez, A.; Kiwi, J.; Water Res. 2000, 34, 582.

4. Lin, S. H.; LO, C. C.; Water Res. 1997, 31, 2050.

5. Kang, S.; Liao, C.; Chen, M.; Chemosphere 2002, 46, 923.

6. Leão, M. M. D.; Carneiro, E. D.; Andrade, L. I. F.; Soares, A. F. S.; Oliveira, A. P.; Santana, D. W. E. A.; Resumos do $20^{\circ}$ Congresso Brasileiro de Engenharia Sanitária e Ambiental, Rio de Janeiro, Brasil, 1999.

7. Kang, Y. W.; Hwang, K.; Water Res. 2000, 34, 2786.

8. Oliveira, D. M.; Dissertação de Mestrado, Universidade Federal de Minas Gerais, Brasil, 2005.

9. Rodrigues, F. S. F.; Dissertação de Mestrado, Universidade Federal do Rio de Janeiro, Brasil, 2004.

10. Ohlweiler, O. A.; Teoria e Prática da Análise Quantitativa Inorgânica, $1^{a}$ ed., Universidade de Brasília: Brasília, 1968.

11. Kang, Y. W.; Cho, M.; Hwang, K.; Water Res. 1999, 33, 1247.

12. AWWA/APHA/WEF; Standard Methods for the Examination of Water and Wastewater, 20 ${ }^{\text {th }}$ ed., Joint Editorial Board: Washington, 1997.

13. Neyens, E.; Baeyens, J.; J. Harzard. Mater. 2003, 28, 33.

14. Menezes, J. C. S.; Pizzolato, T. M.; Schneider, I. A. H.; Quim. Têxtil 2005, 79 . 